\title{
Carbon-Based Electrode Materials for Supercapacitor: Progress, Challenges and Prospective Solutions
}

\author{
Xian Jian, Shiyu Liu, Yuqi Gao, Wei Tian, Zhicheng Jiang, Xiangyun Xiao, Hui Tang and Liangjun Yin \\ Center of Micro-nano Functional Materials and Devices, School of Energy Science and Engineering, University of Electronic \\ Science and Technology of China, Chengdu 611731, China
}

\begin{abstract}
Carbon-based materials are typical and commercially active electrode for supercapacitors due to their advantages such as low cost, good stability and easy availability. In the light of energy storage, supercapacitors mechanism is classified into EDLCs (electrochemical double layer capacitors) and pseudocapacitors. Multidimensional carbon nanomaterials (active carbon, carbon nanotube, graphene, etc.), carbon-based composite and corresponding electrolyte are the critical and important factor in the eyes of researcher. In this minireview, we will discuss the storage mechanism and summarize recent developed novel carbon and carbon-based materials in supercapacitors. The techniques to design the novel nanostructure and high performance electrodematerials that facilitate charge transfer to achieve high energy and power densities will also be discussed.
\end{abstract}

Key words: Carbon materials, storage mechanism, electrochemical double layer capacitors, pseudocapacitors.

\section{Introduction}

In terms of energy storage devices, there are three main electrochemical systems, namely, battery, EC (electrochemical capacitor) and capacitor [1]. Differing from conventional capacitors and batteries, ECs (also called supercapacitors, SC) are highly valued by researchers since their good performance in delivering significant energy in the high-power or pulse-power forms. Specifically, SCs can instantaneously provide relatively higher power density $(15 \mathrm{~kW} / \mathrm{kg}$ ) than batteries (e.g. up to $1 \mathrm{~kW} / \mathrm{kg}$ for Li-ion battery), and higher energy density ( $5 \mathrm{Wh} / \mathrm{kg}$; Conway 1999) than conventional dielectric capacitors, but lower energy density than Li-ion battery (180 $\mathrm{Wh} / \mathrm{kg}$ ). Such excellent properties make SC promising energy storage devices in many applications including hybrid electric vehicles, consumer electronics, memory back-up systems and industrial power and energy management [2], but still the challenge of lower energy

Corresponding author: Xian Jian, associate professor, $\mathrm{Ph} . D$., research fields: carbon nanomaterials, energy materials, microwave absorption. E-mails: jianxian@uestc.edu.cn (Xian Jian), tanghui@uestc.edu.cn (Hui Tang) and ljyin@uestc.edu.cn (Liangjun Yin). density and longer cycle-life remains to be addressed.

The related energy storage performance is mainly attributed to two kinds of approaches, namely, electric double-layer capacitance and a pseudocapacitive contribution of quick faradaic reactions resulting from surface functionalities at the electrode/electrolyte interface. The double-layer capacitance is a typical charge storage mechanism and the ability of charge is depended on the surface feature of active materials, charge transmission path and electrolyte properties. The increasing the surface of material, shortening the charge transmission distance and studying high-performance electrolyte are recent typical research interests. Besides, for the pseudocapacitive contribution in capacitor, the polarization of surface functional groups on corresponding active material plays a positive role in terms of enhancing the surface wettability in aqueous electrolyte and decreases the resistance. However, the faradaic reactions of these functional groups are often limited and unstable during long-term cycling.

At present, carbon materials including ACs (activated carbons), CNTs (carbon nanotubes) and carbon-based composites and hybrids are developed as 
active materials in SC. Here we summarized the related mechanism, recent progress and challenges.

\section{Supercapacitor Types and Corresponding Energy Storage Mechanisms}

With respect to the sorts of electrode materials, SCs can be divided into three classes: carbon-based SCs, metal oxides SCs, and conductive polymers SCs. Initially, we make a short summary for the metal oxides capacitors and conductive polymers capacitors. As an important candidate material for SC, transition metal oxides have been aroused a great attention because of their high specific capacitance coupled with very low resistance resulting in a high specific power, which makes them very appealing in commercial applications[3]. In the early research, $\mathrm{RuO}_{2}$ is a promising SC material due to its high specific capacitance, long cycles life, high conductivity, good electrochemical reversibility and high rate capability. But $\mathrm{RuO}_{2}$ meets the challenge of high cost and barren reveres. Therefore, researchers consider other alternatives including $\mathrm{MnO}_{2}, \mathrm{Co}_{3} \mathrm{O}_{4}, \mathrm{NiO}, \mathrm{WC}, \mathrm{V}_{2} \mathrm{O}_{5}$, $\mathrm{Fe}_{3} \mathrm{O}_{4}$, etc. for $\mathrm{SC}$ active materials. The porous and novel nanostructures for these materials have been paid attention to improve the specific surface, stability and electrochemical performance. At present, most of the single-component metal oxides have the specific capacitance about $600 \mathrm{~F} / \mathrm{g}$, this low performance hinders their practical application. Moreover, some hybrid metal oxides, hydroxide and compound are developed such as amorphous $\mathrm{Ru}_{1-\mathrm{y}} \mathrm{Cr}_{\mathrm{y}} \mathrm{O}_{2} / \mathrm{TiO}_{2}(1,272$ $\mathrm{F} / \mathrm{g})$ [4], $\mathrm{Mn}_{3} \mathrm{O}_{4}-\mathrm{NiO}-\mathrm{Co}_{3} \mathrm{O}_{4}(7,404 \mathrm{~F} / \mathrm{g})$ [5], $\mathrm{Ni}(\mathrm{OH})_{2}$ $(578 \mathrm{~F} / \mathrm{g}), \mathrm{Cl}$-doped carbonated cobalthydroxide $\left(\mathrm{Co}\left(\mathrm{CO}_{3}\right)_{0.35} \mathrm{Cl}_{0.20}(\mathrm{OH})_{1.10}\right) \quad \mathrm{NWs} \quad$ (nanowires) $(9893.75 \mathrm{~F} / \mathrm{g}$ at $0.5 \mathrm{~A} / \mathrm{g})$ [6]. These founding enrich the SC materials and are in light of the designing concept.

Simultaneously, researchers also develop another kind of SC active material using CPs (conducting polymers), due to their low cost, low environmental impact, high conductivity in a doped state, high voltage window, high storage capacity, porosity/reversibility, and adjustable redox activity through chemical modification [7]. CPs possess the structure of the $\pi$-conjugated polymer chains and have the feature of the fast and reversible oxidation and reduction processes. The redox reactions in the CPs happen throughout their entire materials, not just on the surface due to the reason that ions are easily transferred/released from the polymer backbone into the electrolyte when oxidation/reduction takes place. CPs can be positively and negatively charged with ion insertion in the polymer matrix to be "p-doped" and "n-doped" polymers, respectively. CPs can be classified into three major catteries: symmetric "p-p", asymmetric " $p-p$ "” and asymmetric " $n-p$ "' types. The common electrical CPs in SC applications are PANI (polyaniline), PPy (polypyrrol), PTh (polythiophene), and their corresponding derivatives. Recently a lot of CPs-based composites are also developed such as PANI-MWNTs, PPY-graphene, PPY-RuO ${ }_{2}$, PANI-Ti, PANI-Si, etc.

For the advanced SC in practical application, four parameters including energy density, power density, voltage window and cycle life are very significant. However, the improvement of operation voltage and higher energy is often accompanied by sacrificing power density and cycle life. Various active materials were investigated to address these issues, for instance, oxides, conducting polymer, carbon and their hybrids. In most of commercialized system, the SCs are often used carbon materials (about 95\%) as the active material due to its advantages such as good conductivity, low cost and high surface area, thus we will discuss SCs using carbon related materials as the active electrode materials.

SCs as a kind of new energy storage device have different energy storage mechanism with conventional capacitors. According to the different energy storage mechanism, SCs ought to be classified into three types: EDLCs (electrochemical double layer capacitors), pseudocapacitors and the compound of two types above (Fig. 1) [8]. EDLCs mainly rely on the electrical 
A

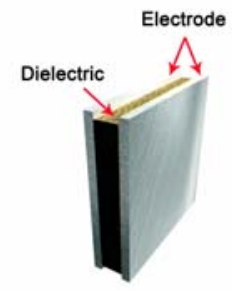

Electrostatic capacitor
B

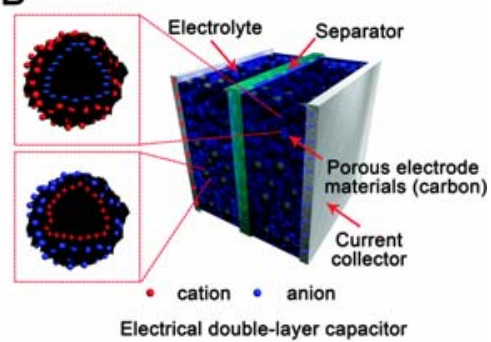

D

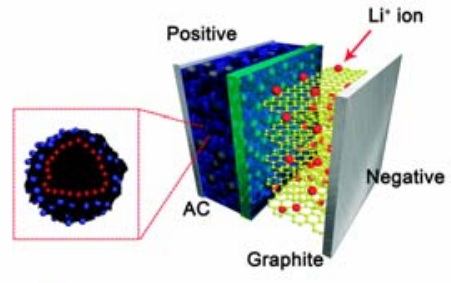

- anion Lithium ion capacitor

Fig. 1 Schematic representation of (A) electrostatic capacitor and three SCs: (B) EDLCs; (C) pseudocapacitors; (D) hybrid capacitor. Reprinted with permission from Ref. [8] Copyright (2015) Royal Society of Chemistry.

double layer effect which reserves static charges on the interface of electrodes and electrolytes. Pseudocapacitors bear fast and reversible faradaic redox reactions at the surface of active materials. Because of faradaic redox reactions, the cycle lives of pseudocapacitors are lower than the former. In general, the active materials of pseudocapacitors are those with high energy density, facilitating pseudocapacitors' better electrochemical performance. The novel nanostructure and type for the developing active materials are summarized in the following section.

\section{Recent Progress}

\subsection{Carbon-Based Electrodes for EDLC}

Carbon-based materials have attracted much attention for their desirable chemical performance and physical characteristics according to the principle of EDLC. And the capacitance in EDLS is depended on an electrode-potential-dependent accumulation of electrostatic charge at the interface [7]. The mechanism of surface electrode charge generation includes surface dissociation as well as ion adsorption from both the electrolyte and crystal lattice defects. It can be seen from Fig. 1b that charges can be stored and separated at the interface between the conductive active materials (such as carbon particles or metal oxide particles) and the electrolyte. This interface can be treated as a capacitor with an EDLC, which can be expressed as Eq. (1):

$$
C=\frac{A \varepsilon}{4 \pi d}
$$

where $A$ is the area of the electrode surface, $\varepsilon$ is the medium (electrolyte) dielectric constant and $d$ is the effective thickness of the electrical double layer. Many researchers focus on the surface improvement of active materials with porous structure and alternative electrolyte with high dielectric constant and feasible charge transport path. Therefore, further improving the ion transport rate of carbon electrodes without sacrificing the charge storage performance is particularly important for EDLCs. Herein, the ion transport kinetics is defined by the ion transport time as Eq. (2) $[9,10]$ :

$$
\tau=L^{2} / D
$$

where $L$ refers to the ion transport length and $D$ refers to the ion transport coefficient. Two dimensional (2D) carbon constitutes are better alternative because they 
possess intrinsic advantage over particulate activated carbon since the ion transport length is dramatically shortened in the thin dimension. Bing et al. [10] developed a two-step etching route to ultrathin carbon nanosheets for high performance electrical double layer capacitors with specific capacitance of $220 \mathrm{~F} / \mathrm{g}$ at $0.5 \mathrm{~A} / \mathrm{g}$.

Niu et al. [12] used multi-walled carbon nanotubes as the electrode substrate materials, performing the specific capacitance $(102 \mathrm{~F} / \mathrm{g})$ and power density $(8$ $\mathrm{kW} / \mathrm{kg}$ ). An et al. [13] discovered the single-walled carbon nanotubes, exhibiting the specific capacitance $(180 \mathrm{~F} / \mathrm{g})$, energy density $(20 \mathrm{~W} / \mathrm{kg})$ and power density $(7 \mathrm{~kW} / \mathrm{kg})$. Futaba [14] reported an ordered single-walled carbon nanotubes, indicating that single-walled carbon nanotubes with ordered array, since the neat structure provides more channels for the movement of ions. Comparing with AC, this special structure of CNT allows more excellent conductivity and chemical performance. However, the existence of large contact resistance between the electrode material and electronic collector greatly restricts the power performance, which brings the growth of carbon nanotubes on conductive substrate (such as gold) in order to decline this contact resistance. Furthermore, the composite of conducting polymer and CNT is an effective approach to reduce the contact resistance and enrich the synergic effect resulting in the improvement of SC performance. For example, the composite of PANI-20 wt $\%$ MWNTs has the higher specific capacitance up to $670 \mathrm{~F} / \mathrm{g}$ in $1 \mathrm{M} \mathrm{H}_{2} \mathrm{SO}_{4}$ [7].

Graphene is a single-layer, mesh honeycomb construct composed of carbon atoms (Fig. 2). In 2004, British scientist Geim et al. [15] first reported the discovery of graphene indicating that graphene is not only the thinnest material but also exhibits extraordinary electronic properties within 2D flat structure. A realistic quantum capacitance reported by Paula et al. using aryldiazonium modified large area few-layer graphene as electrodes [16]. In addition to its unusual electronic properties, graphene has many excellent properties. Graphene structure can be described as a huge aromatic hydrocarbon-based polymer, demonstrating very excellent electrical and thermal conductivity with 2D flat structure. Since the nanotubes can be considered to be curled graphene prepared, it indicates the pretty mechanical properties of graphene as well as that of carbon nanotubes. Recently, Adriano et al. [17] developed a facile electrochemically exfoliated method to produce

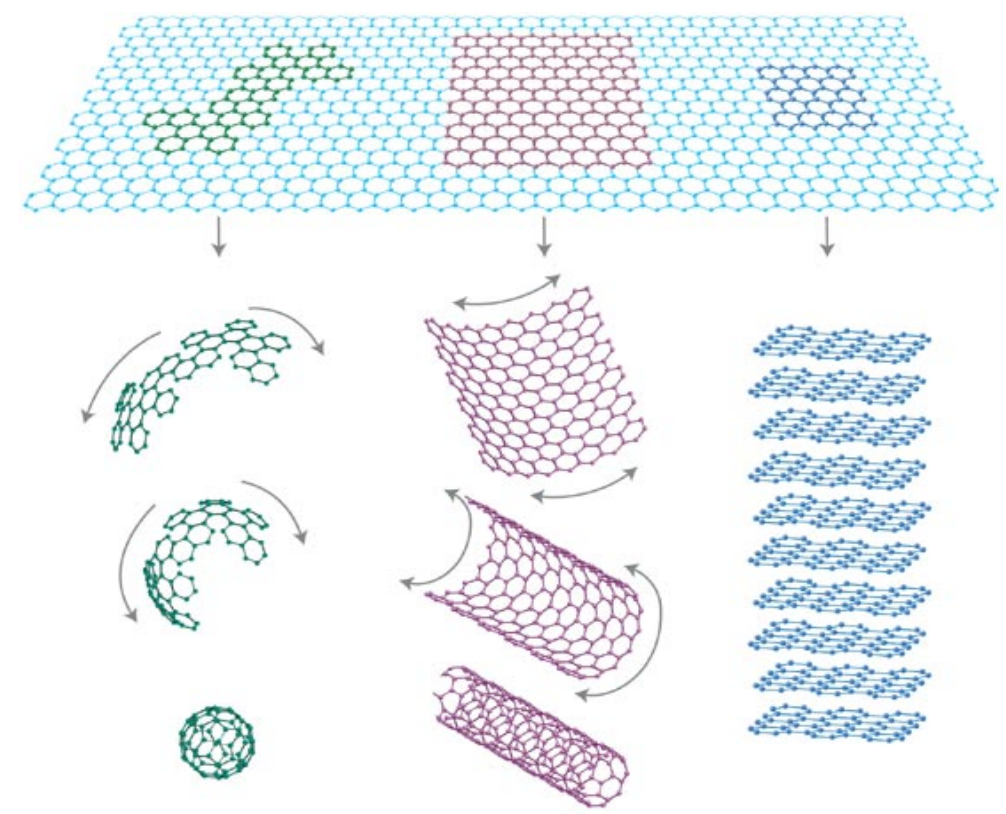

Fig. 2 Graphene can be wrapped up into 0D buckyballs, rolled into 1D NTs or stacked into 3D graphite. Reprinted with permission from Ref. [15] Copyright (2016) Nature Publishing Group. 
graphene and graphene oxide for energy storage by introducing $\mathrm{H}_{2} \mathrm{SO}_{4}, \mathrm{Na}_{2} \mathrm{SO}_{4}$ and $\mathrm{LiClO}_{4}$. It is known that graphene has excellent electrical conductivity, thermal conductivity, good mechanical properties and a large surface area. To take advantage of these unique properties, scientists try to design composite electrode materials with graphene and graphene oxide as substrate.

Besides, other carbon-based materials also have attracted much attention for their desirable chemical performance and physical characteristics according to the principle of EDLC, such as activated carbon, carbon aerogels, carbon nanotubes and graphene. AC (activated carbon) is the early used carbon electrode material among all carbon-based materials due to its abundant resource, lower costs, simple methods of compound and stability. In theory, the larger specific surface area activated carbon possesses the higher specific capacitance. However, the common activated carbon normally displays low specific surface area, which results in low specific capacitor. Moreover, the arrangement of pore could also have an influence on specific capacitor. Thus, improving the specific surface area and distributed pore will push the development of active carbon.

CNF (carbon nanofiber) includes solid and hollow CNF. The structure of $\mathrm{CNF}$ is the result of the convolution of multilayers graphene. The CNF interlayer spacing is greater than that of graphene, so the charge-discharge capacity of CNF displays greater than it generally, and these tubular structures would maintain its stability after many charge and discharge cycles. Tao et al. [18] prepared multi-branched porous carbon nanofibers doped with $\mathrm{Li}, \mathrm{Na}$, or $\mathrm{K}$ and applied it in electrochemical double-layer capacitor with a specific capacitance of ca. $297 \mathrm{~F} / \mathrm{g}$ was obtained using $6 \mathrm{M} \mathrm{KOH}$ as the electrolyte. The porous structure of CNF benefits the ability to store charge and enrich the channel for ion transferring. Meanwhile, nitrogen-doped porous CNF webs were also been developed by Huang group, these CNFWs (CNF webs) were prepared by carbonization-activation of polypyrrole nanofiber webs with $\mathrm{KOH}$, and CNFWs exhibit a superhigh reversible capacity of $943 \mathrm{mAh} / \mathrm{g}$ at a current density of $2 \mathrm{~A} / \mathrm{g}$ even after 600 cycles, which is ascribed to the novel porous nanostructure and high-level nitrogen doping [19].

Besides, helical carbon nanofibers (also named carbon nanocoil, CNC) are one of novel carbon nanostructures with higher surface area, super elasticity, and good stability. Recently, Izumi et al. [20] manufactured EDLC using carbon nanocoils and evaluated it at a high scan rate shown in Fig. 3. They compared carbon nanomaterials such as AC (activated carbon), AcB (arc-black) and carbon nanocoil by their specific capacitances. $\mathrm{CNC}$ and $\mathrm{AcB}$ showed the lower charge transfer resistance than $\mathrm{AC}$ in the measurement of EIS (electrochemical impedance spectroscopy), and the EDLCs using CNC and AcB kept their specific capacitance almost the same even at a high scan rate.

CAG (carbon aerogels) actually belongs to an ideal carbon-based material for electrical double layer capacitors because of multiple pore structure, equal pore size (less than $50 \mathrm{~nm}$ ) and high specific surface
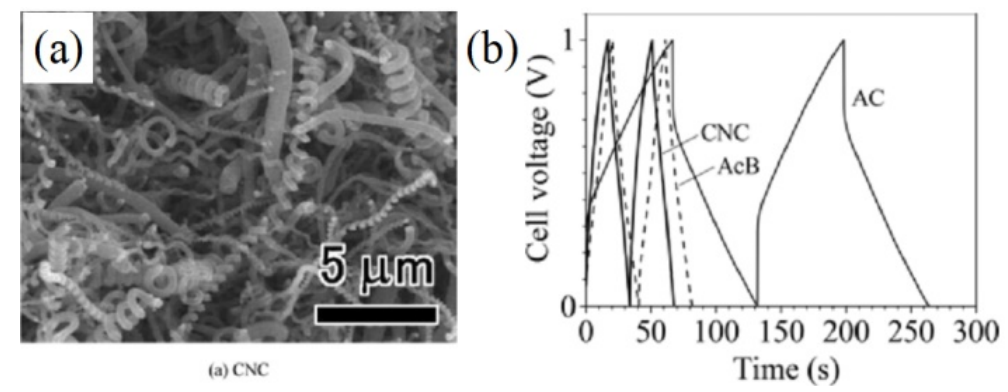

Fig. 3 SEM image of carbon nanocoils and charge/discharge curves of $\mathrm{CNC}$, AcB and AC. Reprinted with permission from Ref. [20] Copyright (2016) Wiley. 
area $\left(600 \sim 1,000 \mathrm{~m}^{2} / \mathrm{g}\right)$. Recently, Liu et al. [21] have reported an active aerogels, which displays high specific capacitance $(250 \mathrm{~F} / \mathrm{g})$. But a major drawback of the preparation of carbon aerogels is that, it is difficult to be industrialized. Doping and exploration of new electrolyte are also effective approaches to improve the performance. Liu et al. [22] developed the NCMF (nitrogen-doped carbon microfiber) with an excellent capacitance of $196 \mathrm{Fg}^{-1}$ at scan rate of 5 $\mathrm{mV} \mathrm{s}^{-1}$. More importantly, a high energy density $\left(\approx 22.7 \mu \mathrm{Wh} \mathrm{cm}^{-2}\right)$ and power density $(\approx 10.26$ $\mathrm{mW} \mathrm{cm}^{-2}$ ) are achieved by the all-solid-state SC based on NCMF. Moreover, Yang et al. [23] prepared pore size-controlled carbon aerogels for EDLC electrodes in organic electrolytes (1 $\mathrm{M}$ tetraethylammonium tetrafluoroborate in acetonitrile, $\mathrm{TEABF}_{4} / \mathrm{ACN}$ ). Pore size-controlled carbon aerogels were obtained from resorcinol and formaldehyde by varying polymerization time. The results provide an effective way to design the optimum pore size in determining its electrochemical behavior. Except for the hydrolysis and organic electrolyte, the ionic liquid is also considered to prove the electrochemical potential window of the electrolyte used in the energy storage devices. Liew et al. [24] report the investigation of ionic liquid-doped ion conducting polymer electrolytes for carbon-based EDLCs (electric double layer capacitors), and they get higher specific capacitance in EDLC comprising ionic liquid-doped polymer electrolytes. Ionic liquid-based poly(vinyl alcohol)/ammonium acetate $\left(\mathrm{CH}_{3} \mathrm{COONH}_{4}\right)$ ion conducting polymer electrolytes are prepared by solution casting technique.

During the preparation process of energy materials, it will consume a lot of energy and lead to environmental pollution in some degree. Therefore, the use of the original biomass is a sustainable way of development. Recently, Wang et al. [25] reported a cheese-like porous carbon obtained from Lignin, an abundant biomass constituent in nature. EDLC fabricated using this $\mathrm{AC}$ as an electrode material showed a specific capacitance of 248 and $211 \mathrm{~F} / \mathrm{g}$ at a low current density of $0.1 \mathrm{~A} / \mathrm{g}$ and $10 \mathrm{~A} / \mathrm{g}$ in $6 \mathrm{M} \mathrm{KOH}$, respectively. This work demonstrates that the $\mathrm{N}$-doped cheese-like porous AC is a promising electrode material for electric double layer capacitors.

Summarily, carbon-based electrodes for EDLC are focused on the field of preparation technology for novel carbon structure with high surface area and porous microstructure to favor the charge storage and enhance ion diffusivity, and the effective including organic, ionic liquid, etc.

\subsection{Carbon-Based Material for Pseudocapacitors}

Different from the EDLC, the thermodynamics redox reaction accompanied with charge acceptance and potential change results in the formation of derivative capacitance named pseudocapacitance. The essence of the pseudocapacitance is the Faradic in origin, involving fast and reversible redox reactions between the electrolyte and electro-active materials on the electrode surface [2]. Compared with EDL capacitance, pseudo-capacitance can be higher than EDL capacitance because of additional contribution from the charge receiving and departing associated with redox action. Normally, the superficial redox transitions of electrode active species involve proton and electron double injection/expulsion. For example $\mathrm{RuO}_{2}$, the proton and electron movement can be described as the following Eq. (3):

$$
\mathrm{RuO}_{x}(\mathrm{OH})_{y}+\delta \mathrm{H}^{+}+\delta \mathrm{e}^{-} \Leftrightarrow \mathrm{RuO}_{x-\delta}(\mathrm{OH})_{y+\delta}
$$
where $\mathrm{RuO}_{\mathrm{x}}(\mathrm{OH})_{\mathrm{y}}$ and $\mathrm{RuO}_{\mathrm{x}-\delta}(\mathrm{OH})_{\mathrm{y}+\delta}$ represent the interfacial oxyruthenium species at higher and lower oxidation states [2]. In light of Eq. (3), the reversible redox transitions depend on both proton exchange and electron-hopping processes. Then under a proton-rich electrolyte environment (e.g. $\mathrm{H}_{2} \mathrm{SO}_{4}$ ), the faradic charges can be reversibly stored and delivered through the redox transitions of active materials. Recently, Mai et al. [26] make use of $\mathrm{Cu}^{2+}$ reduction with carbon-oxygen surface groups of the binder-free electrode in a new redox-active electrolyte and 
corresponding synergistic interaction between redox-active electrolyte and binder-free functionalized carbon to get ultrahigh SC performance with an enhancement in the voltammetric capacitance of 4,700 $\mathrm{F} / \mathrm{g}$. Besides, the feasibility environment for electron and ion delivery is also considered by researcher. For instance, the hydrous ruthenium oxide $\left(\mathrm{RuO}_{2} \cdot \mathrm{xH}_{2} \mathrm{O}\right)$ exhibited a much higher specific capacitance $(720 \mathrm{~F} / \mathrm{g})$ than anhydrous ruthenium oxide $\left(\mathrm{RuO}_{2}\right)$. Besides, Mahmood et al. [6] developed a well-defined and self-stabilized chlorine-doped carbonated cobalthydroxide $\quad\left(\mathrm{Co}\left(\mathrm{CO}_{3}\right)_{0.35} \mathrm{Cl}_{0.20}(\mathrm{OH})_{1.10}\right) \quad \mathrm{NWs}$ (nanowires) via a facile hydrothermal method for SCs to introduce the concept of deep Faradaic redox reaction. Excepted for unique composition along with define porosity and existence of structure stabilizer counter anions, hydrophilic nature of this NW makes the deep diffusion of electrolyte ions come true. The NWs present extraordinary capacitance of 9,893.75 F/g at $0.5 \mathrm{~A} / \mathrm{g}$ and excellent energy density of $220 \mathrm{Wh} / \mathrm{kg}$ along with high rate capability and stability for 10,000 cycles. These results have shown that the NWs will become promising candidates for the next generation of energy storage devices for high power applications.
Though numerous efforts have been focused on fabricating nanostructured materials such as porous structure that provided an effective pathway for electrolyte and electron transportation, but the enhanced surface area becomes less efficient in contributing to the total charge storage based on the Huang's experimental and theoretical study [27]. They proposed an universal model to the understanding of the relationship between the electrolyte ion size and the carbon pore size in trying to assure the optimal pore size needed in electrode active species. In case of the mesoporous structures $(2-50 \mathrm{~nm})$ in carbon-based electrodes, the SC (specific capacitance) normalized to SSA (specific surface area) drops as the pore size decreases (Fig. 4, zone III and zone IV). Therefore the improvement of surface area contributes less to the total charge storage. By assuming mesopores are cylindrical, the specific capacitance with respect to SSA could be written in the form of Eq. (4).

$$
C / A=\frac{\varepsilon_{r} \varepsilon_{0}}{b \ln \left(\frac{b}{b-d}\right)}
$$

where $\varepsilon_{\mathrm{r}}$ is the electrolyte dielectric constant, $\varepsilon_{0}$ is the dielectric constant of the vacuum, $b$ is the pore radius and $d$ is the distance between approaching electrolyte

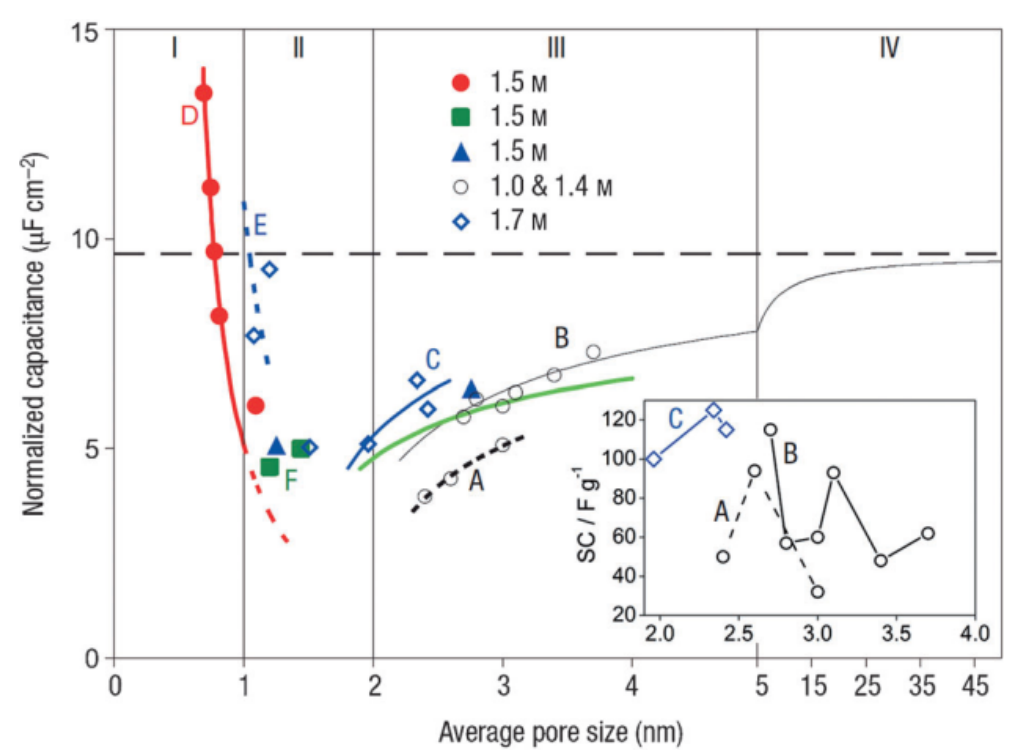

Fig. 4 Specific capacitance normalized by SSA as a function of pore size for carbon materials including templated microporous carbon ( $A$ and $B$ ), activated mesoporous carbon (C), microporous carbide-derived carbon (D and F), and microporous activated carbon (E), are presented. Inset: Specific capacitance normalized by electrode mass as a function of mesopore size. Reprinted with permission from Ref. [28] Copyright (2013) John Wiley \& Sons Inc. 
ion and the carbon surface. In case of less than $1 \mathrm{~nm}$ pore size, the ion adsorption could be described by an "electrical wire in cylinder" model as presented in the Eq. (5).

$$
C / A=\frac{\varepsilon_{r} \varepsilon_{0}}{b \ln \left(\frac{b}{a_{0}}\right)}
$$

However, little report has addressed the complex relationship between the texture parameters of the electrode materials and the supercapacitance of ECs. Recently, Aiguo Hu group developed a new model to simulate the accommodation of the solvated ions at the electrode surface [29]. Fig. 5 shows their simulation on the theoretical capacitance versus the ratio of the pore and solvated ion diameters $(\mathrm{R} / \mathrm{r})$ in a porous electrode with a fractal dimension $\mathrm{D}$ of 2.5 . In region $\mathrm{I}$, the specific capacitance increases with a decrease in pore size, similar trend reported by Gogotsi et al. in the CDC system. The hosting capacity of a cylinder channel does not considerably change in this region; however, the SSA constantly increases with a decrease in pore size. In region II, the SSA slowly decreases with an increase in pore size; however, the solvated-ion hosting capacity of mesopores sharply increases around $\mathrm{R} / \mathrm{r}=2$, resulting in a rapid increase in specific capacitance. In region III, the SSA steadily decreases with an increase in pore size for porous materials with $\mathrm{D}>2$, whereas the solvated-ion hosting capacity of pores is close to its maximum [29]. This work is very significant to address the competitive and combined effect of solvated-ion hosting capacity and hosting capacity of a cylinder channel on the specific capacitance with changing pore size.

Another route is to design novel and effective hybrid systems where one SC electrode is associated with a faradic electrode in organic or aqueous electrolyte. The carbon-based hybrid with metal oxides $\left(\mathrm{MnO}_{2} / \mathrm{CNT}\right.$ [30], $\mathrm{NiO} /$ graphene, $\mathrm{Co}_{3} \mathrm{O}_{4}$ /graphene, etc.), metals hydroxide $\left(\mathrm{Ni}(\mathrm{OH})_{2} /\right.$ graphene, $\mathrm{Co}(\mathrm{OH})_{2} /$ graphene, etc. $)$ and other multiplex compound $\left(\mathrm{Ni}(\mathrm{OH})_{2}-\mathrm{MnO}_{2}-\mathrm{rGO}\right.$ [31], $\mathrm{CFs} / \mathrm{Co}(\mathrm{OH})_{\mathrm{x}} \mathrm{CO}_{3}$ and $\mathrm{CFs} / \mathrm{Ni}(\mathrm{OH})_{2}$ [32], etc.) are still being extensively investigated at present. As typical electrode active species, $\mathrm{RuO}_{2}$ is the most promising pseudocapacitive electrode material among the transition metals with remarkably high specific capacitance, high chemical and thermal stability, and good electrochemical redox properties, then their hybrid such as $\mathrm{RuO}_{2} /$ reduced grapheme [33], $\mathrm{RuO}_{2} \cdot \mathrm{nH}_{2} \mathrm{O} /$ carbon nano-onions [34], $\mathrm{RuO}_{2} / \mathrm{CNFs}$ [35], etc. are also developed to get reasonable structure and improve their performance. Manjusha group present a sol-gel technique to form homogeneously dispersed $\mathrm{RuO}_{2}$ nanoparticles on CNOs (carbon nanoonions), and then get a flexible substrate in a novel process of modifying a conducting carbon paper delivers
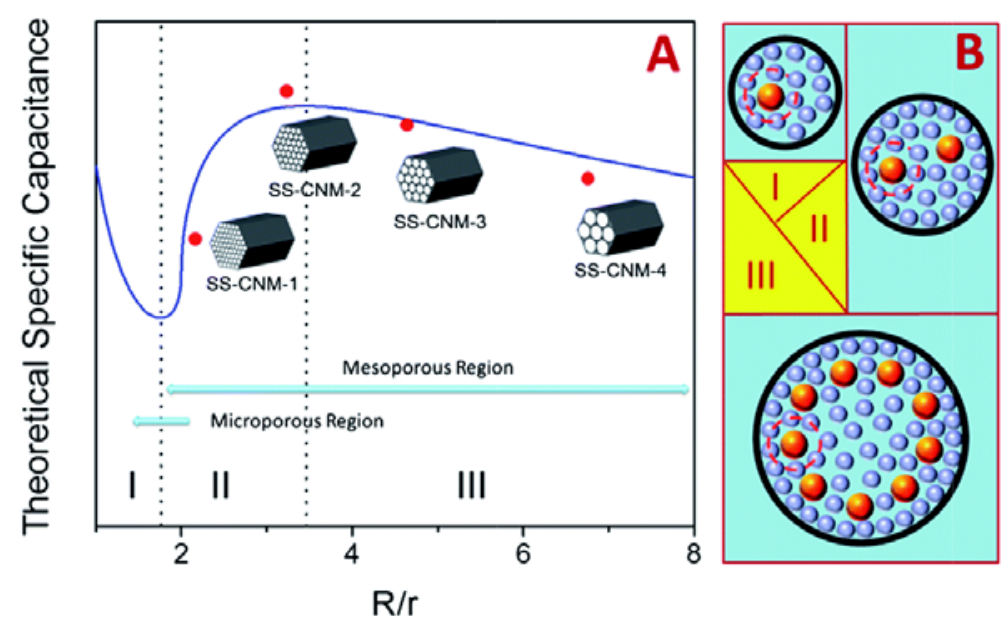

Fig. 5 (A) Fitting the capacitances of the SS-CNMs with the model plot. (B) Drawings of solvated ions residing in pores with $\mathrm{R} / \mathrm{r}$ values of 1.5 (top left), 2 (top right), and 4 (bottom). Reprinted with permission from Ref. [29] Copyright (2014) Royal Society of Chemistry. 
high energy density $(16.2 \mathrm{Wh} / \mathrm{kg})$ even at the power density of 9,885 W/kg as shown in Fig. 6.

Though the $\mathrm{RuO}_{2}$ composite or hybrids possess very attractive properties, researchers also try to address the challenge of high cost and barren reveres by developing other non-toxic and low cost substitutions. Combined the advantages of $\mathrm{Mn}_{\mathrm{x}} \mathrm{O}_{\mathrm{y}}$ and carbon nanostructures, the $\mathrm{MnO}_{2} /$ carbon sphere [36], $\mathrm{MnO}_{2} / \mathrm{CNTs}$ [37], $\mathrm{MnO}_{2}$ nanoneedles/CNT [38], $\mathrm{MnO}_{2}$ /activated carbon [39], $\mathrm{Mn}_{3} \mathrm{O}_{4} / \mathrm{AC}$ [40], etc., were also investigated to good cyclability and environmentally friendly electrolyte. Some typical examples are shown in Fig. 7. Furthermore, the macrospore flexible devices were designed using these $1 \mathrm{D}$ or 2D carbon based materials.
But these materials still have relatively low energy densities owing to the narrower voltage window. Besides, some other $\mathrm{NiO}, \mathrm{Co}_{3} \mathrm{O}_{4}$ and $\mathrm{Fe}_{3} \mathrm{O}_{4}$ hybrid with carbon are also developed in the forms of metal oxide composite with active carbon, CNT, graphene, helical carbon nanocoil, carbon black, etc. Take graphene as an example, composites include monometal oxides of cobalt, nickel, and iron, as well as their binary and ternary oxides. For the $\mathrm{NiO}$ and $\mathrm{Co}_{3} \mathrm{O}_{4}$, many preparation methods have been developed such as hydrothermal, sol-gel, precipitation, microwave, electrosynthesis, sonication, CVD, ALD, etc. [41]. $\mathrm{NiO}$ appears to be an especially promising in $\mathrm{SC}$ because of its high theoretical SC $(2,584 \mathrm{~F} / \mathrm{g}$ within $0.5 \mathrm{~V}$ ) and easy oxidation process. At present, some
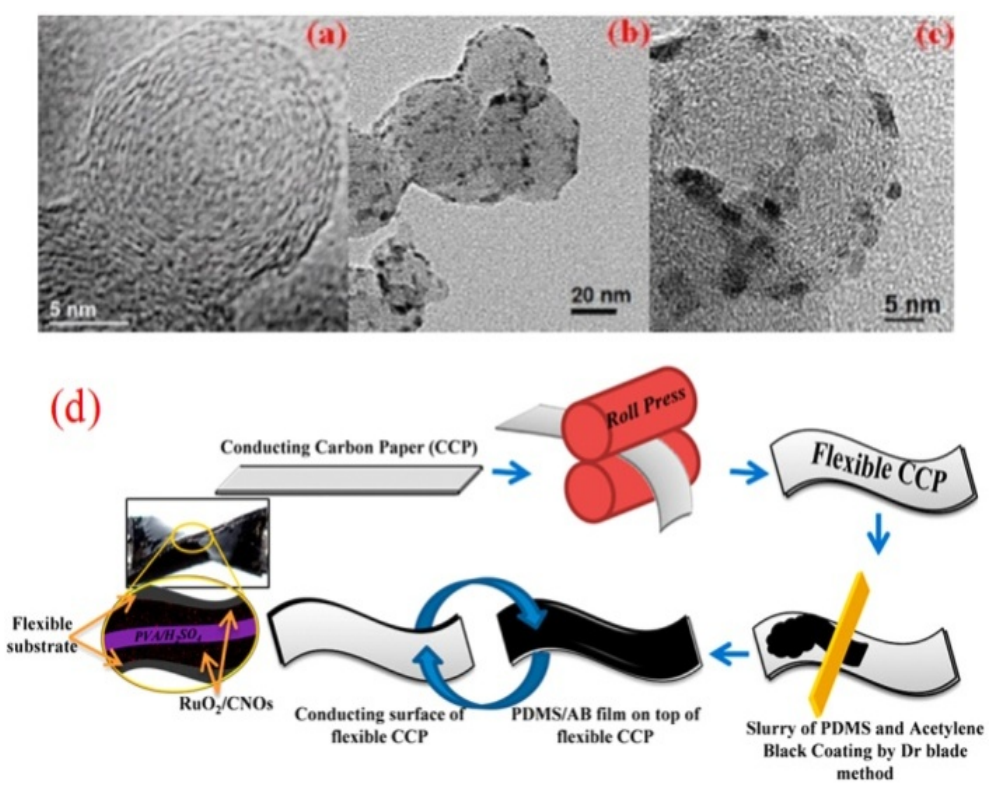

Fig. 6 HRTEM images of (a) CNOs and (b, c) $\mathrm{RuO}_{2}$ decorated on CNOs and (d) schematic illustration of stepwise preparation of flexible conducting substrate and device assembly using $\mathrm{RuO}_{2} / \mathrm{CNOs}$ electrodes. Reprinted from Ref. [34] Copyright (2016) American Chemical Society.

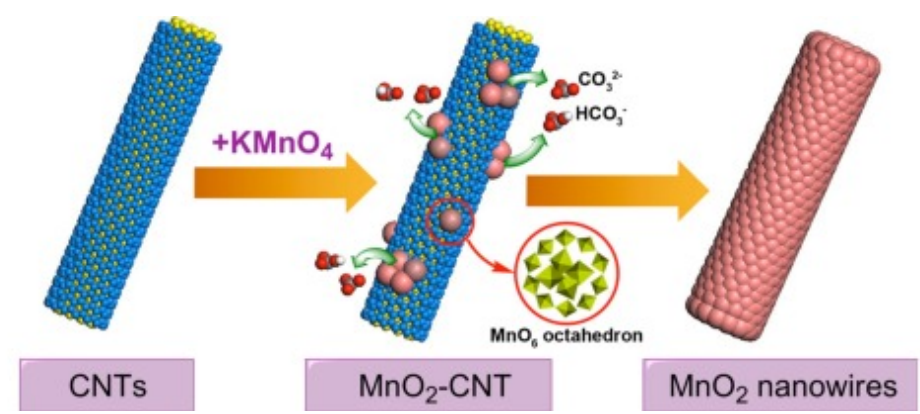

Fig. 7 Schematic illustration for controlled growth of nanostructured $\mathrm{MnO}_{2}$ on CNTs. Reprinted with permission from Ref. [37] Copyright (2015) Elsevier. 
novel structure including nanoflower, nanosheet, nanoparticle, etc. are obtained and applied in the graphene based materials. Yong Qin group use an efficient ALD (atomic layer deposition) method to get the $\mathrm{NiO} / \mathrm{NG}$ composites obtained exhibiting excellent specific capacitance of up to $\sim 1,005.8 \mathrm{~F} / \mathrm{g}$ per mass of the composite electrode (the specific capacitance value is up to $\sim 1,897.1 \mathrm{~F} / \mathrm{g}$ based on the active mass of $\mathrm{NiO}$ ), and stable performance after 1,500 cycles [42]. Meanwhile, the synergetic effects coming from the binary or ternary oxides and carbon matrix favor the further enhancement of SC performance. Some novel composite such as $\mathrm{Fe}_{2} \mathrm{O}_{3} @ \mathrm{MnO}_{2} @ 3 \mathrm{D}$ macroporous graphene, $\mathrm{LiFePO}_{4}-\mathrm{xNy}$ @ MCFs, nickel hydroxide CNTs-CPs [43], etc., are reported to be effective to combine the advantages of carbon and other electrode active metals (metal oxides, conducting plymers, etc.). Jiang et al. [43] developed a ternary hybrid of amorphous nickel hydroxide-carbon nanotube-conducting polymer for SCs with high energy density shown in Fig. 8. The introduction of conductive multiwalled CNTs wrapped with polymer (poly(3,4-ethylenedioxythiophene)-poly(styrenesulfon ate)) is an reasonable method to make up for shortcoming of low conductivity of $\mathrm{Ni}(\mathrm{OH})_{2}$. The reported composite have ultrahigh specific capacitance of $3,262 \mathrm{~F} / \mathrm{g}$ at $5 \mathrm{mV} / \mathrm{s}$ and excellent rate capability $(71.9 \%$ capacitance retention at $100 \mathrm{mV} / \mathrm{s})$ and high energy density of $58.5 \mathrm{Wh} / \mathrm{kg}$ at the power density of $780 \mathrm{~W} / \mathrm{kg}$ as well as long cycle life $(86 \%$ capacitance retention after 30,000 cycles).

Element doping is also an effective way to tune the microstructure and band structure of carbon materials. It is well known that porous nanostructures with large surface areas are typically employed for electrical double-layer capacitors to improve energy storage capacity; however, high surface area carbon-based electrodes result in poor volumetric capacitance because of the low packing density of porous materials. Recently, Faming Gao group reported a fluorine and nitrogen co-doped carbon microspheres with ultrahigh volumetric capacitance of $521 \mathrm{~F} / \mathrm{cm}^{3}$ in aqueous electrolytes and cyclic stability without capacitance loss after 10,000 cycles shown in Fig. 9 [44]. They introduce the active center (fluorine doping as electron acceptors and nitrogen doping as electron-donor) with an acidic character to enrich the pore structure (less than $1 \mathrm{~nm}$ ) and enhance the electrochemistry behaving and properties.

Recently, Meifang Zhu group developed a simple and scalable wet-spinning method for hierarchically structured $\mathrm{MnO}_{2}$ nanowire/graphene hybrid fibers [45]. The hybrid fibers has been improved with a high volumetric capacitance $\left(66.1 \mathrm{~F} \cdot \mathrm{cm}^{-3}\right.$, normalized by the total volume of two fiber electrodes), excellent cycling stability $(96 \%$ capacitance retention over 10,000 cycles $)$, high energy and power density $\left(5.8 \mathrm{mWh} \cdot \mathrm{cm}^{-3}\right.$ and $0.51 \mathrm{~W} \cdot \mathrm{cm}^{-3}$, respectively). Then the hybrid technology is an effective way to get flexible solid-state SCs.
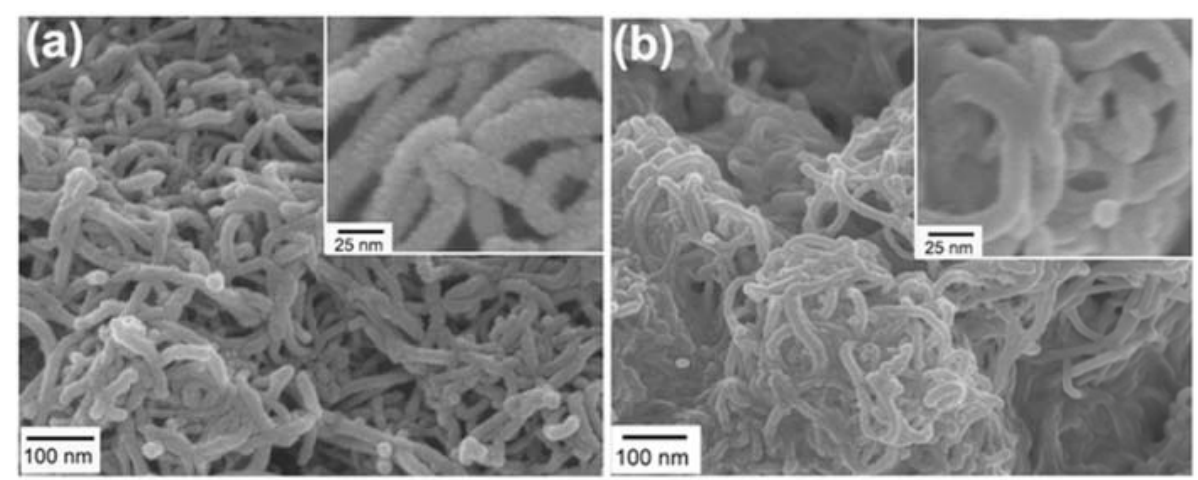

Fig. 8 SEM images of (a) MWCNT/amor-Ni(OH) $)_{2}$ and (b) MWCNT/amor-Ni(OH) $)_{2} / \mathrm{PEDOT}$ :PSS. Reprinted with permission from Ref. [43] Copyright (2015) from Wiley. 

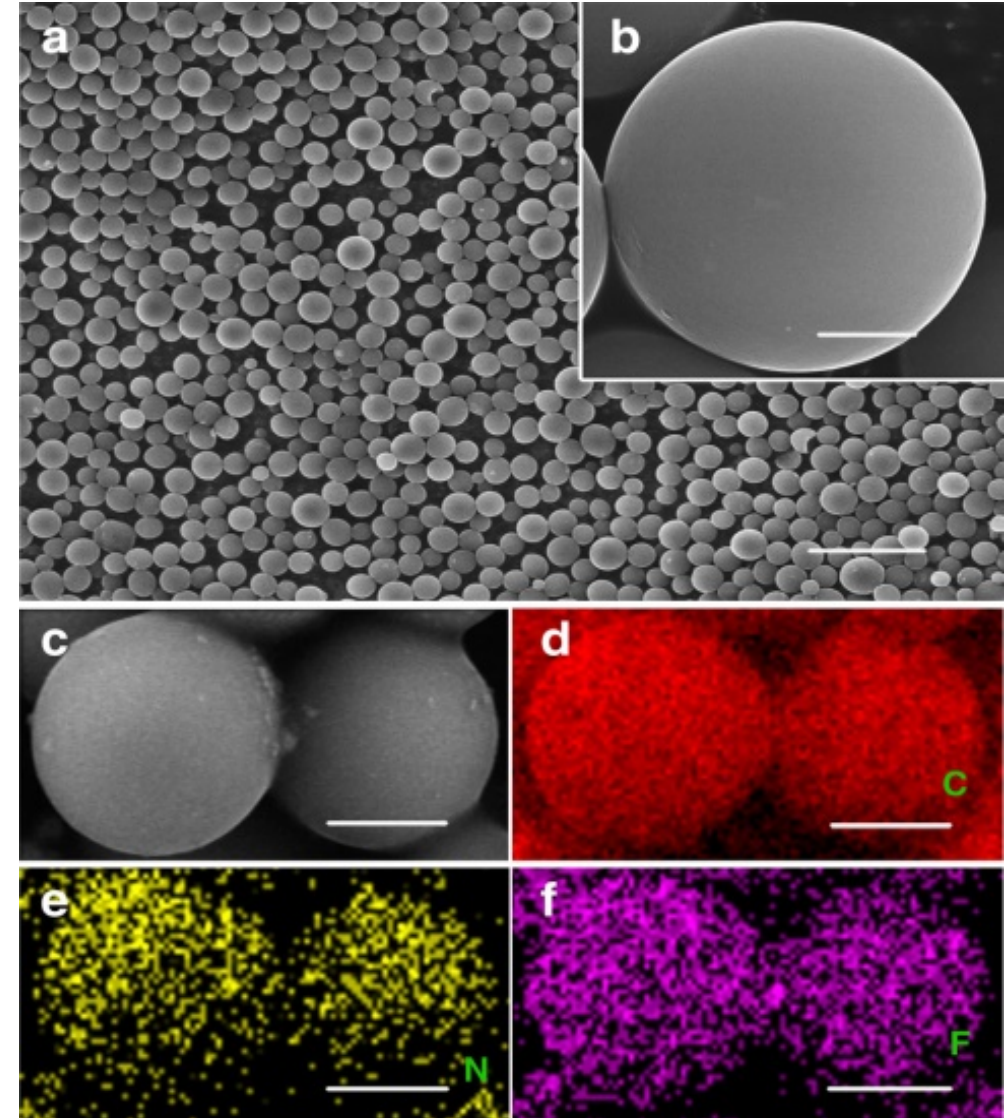

Fig. 9 (a) Lowand (b) high-magnification SEM images of CM-NF, (c) CM-NFs, and EDS elemental mappings of (d) C (red); (e) N (yellow); and (f) F (purple). Scale bar, $15 \mathrm{~mm}$ (a), $1 \mathrm{~mm}$ (b) and $2 \mathrm{~mm}$ (c-f). Reprinted from Ref. [44].

\section{Conclusion and Future Prospective}

We presented a mini review of development, related effects and storage mechanism for the SCs that originate from a combination of pure carbon and carbon-based material. This report mentioning storage mechanism including the EDLC and pseudocapacitor is in light of the materials design to assurance their superior capacitive performances over single component including pure carbon, metal oxides, or simple mixtures thereof. The current research outcomes emphasize the importance of effective morphology, porous structure, surface area and uniformity of active materials such as the distribution of metal oxides on the surface of graphene in hybrid electrode materials.

Some research directions are suggested to improve the SC performance as follows: (1) Hybrid material is a good choice to address the effective synergistic effect to enhance the charge storage and good cycling performance; the method also benefits it strong interaction allowing mechanical stability; (2) Design reasonable porous structure with suitable pore-size distribution and pore length for facilitating ions diffusion at high rate. And this optimal structure should avoid the high surface area result in the poor volumetric capacitance; (3) Effective novel electrolyte provides faster kinetic and more efficient charge and ions transmission, adsorption and release.

\section{Acknowledgment}

This work was financially supported by the National Natural Science Foundation of China (No. 51402040), China Postdoctoral Science Foundation (2015M582539), Science and Technology Support Program of Sichuan Province (2016RZ0054) and the National Hi-Tech Research and Development Program (863 Program) of China (No. 2015AA034202). 


\section{References}

[1] Simon, P., and Gogotsi, Y. 2010. "Charge Storage Mechanism in Nanoporous Carbons and Its Consequence for Electrical Double Layer Capacitors.” Philosophical Transactions of the Royal Society of London A: Mathematical, Physical and Engineering Sciences 368 (1923): 3457-67.

[2] Zhang, L. L., and Zhao, X. S. 2009. "Carbon-Based Materials as Supercapacitor Electrodes.” Chemical Society Reviews 38 (9): 2520-31.

[3] Zhang, Y. et al. 2009. "Progress of Electrochemical Capacitor Electrode Materials: A Review.” International Journal of Hydrogen Energy 34 (11): 4889-99.

[4] Bo, G. et al. 2006. "Amorphous $\mathrm{Ru}_{1-\mathrm{y}} \mathrm{Cr}_{\mathrm{y}} \mathrm{O}_{2}$ Loaded on $\mathrm{TiO}_{2}$ Nanotubes for Electrochemical Capacitors." Electrochimica Acta 52 (3): 1028-32.

[5] Rusi, and Majid, S. R. 2015. "Electrodeposited $\mathrm{Mn}_{3} \mathrm{O}_{4}-\mathrm{NiO}-\mathrm{Co}_{3} \mathrm{O}_{4}$ as a Composite Electrode Material for Electrochemical Capacitor." Electrochimica Acta 175: 193-201.

[6] Mahmood, N. et al. 2015. "Chlorine-Doped Carbonated Cobalt Hydroxide for Supercapacitors with Enormously High Pseudocapacitive Performance and Energy Density." Nano Energy 11: 267-76.

[7] Wang, G., Zhang, L., and Zhang, J. 2012. "A Review of Electrode Materials for Electrochemical Supercapacitors." Chemical Society Reviews 41 (2): 797-828.

[8] Zhong, C. et al. 2015. "A Review of Electrolyte Materials and Compositions for Electrochemical Supercapacitors." Chemical Society Reviews 44 (21): 7484-539.

[9] Wang, H. et al. 2013. "Interconnected Carbon Nanosheets Derived from Hemp for Ultrafast Supercapacitors with High Energy." ACS Nano 7 (6): 5131-41.

[10] Ding, B. et al. 2016. "A Two-Step Etching Route to Ultrathin Carbon Nanosheets for High Performance Electrical Double Layer Capacitors." Nanoscale 8 (21): 11136-42.

[11] Pan, H., Li, J., and Feng, Y. 2010. "Carbon Nanotubes for Supercapacitor." Nanoscale Research Letters 5 (3): 654-68.

[12] Niu, C. et al. 1997. "High Power Electrochemical Capacitors Based on Carbon Nanotube Electrodes." Applied Physics Letters 70 (11): 1480-2.

[13] An, K. H. et al. 2001. "Electrochemical Properties of High-Power Supercapacitors Using Single-Walled Carbon Nanotube Electrodes." Advanced Functional Materials 11 (5): 387-92.

[14] Futaba, D. N. et al. 2006. "Shape-Engineerable and highly Densely Packed Single-Walled Carbon Nanotubes and Their Application as Super-Capacitor Electrodes." Nature Materials 5 (12): 987-94.
[15] Geim, A. K., and Novoselov, K. S. 2007. "The Rise of Graphene." Nat Mater 6 (3): 183-91.

[16] Brooksby, P. A. et al. 2015. "Quantum Capacitance of Aryldiazonium Modified Large Area Few-Layer Graphene Electrodes." The Journal of Physical Chemistry C 119 (46): 25778-85.

[17] Ambrosi, A., and Pumera, M. 2016. "Electrochemically Exfoliated Graphene and Graphene Oxide for Energy Storage and Electrochemistry Applications." Chemistry A European Journal 22 (1): 153-9.

[18] Tao, X. Y. et al. 2006. "Synthesis of Multi-branched Porous Carbon Nanofibers and Their Application in Electrochemical Double-Layer Capacitors." Carbon 44 (8): 1425-8.

[19] Qie, L. et al. 2012. "Nitrogen-Doped Porous Carbon Nanofiber Webs as Anodes for Lithium Ion Batteries with a Superhigh Capacity and Rate Capability." Advanced Materials 24 (15): 2047-50.

[20] Izumi, H. et al. 2016. "Manufacturing of Electric Double-Layer Capacitors Using Carbon Nanocoils and Evaluation of Their Specific Capacitances at a High Scan Rate." Electronics and Communications in Japan 99 (5): 3-10.

[21] Liu, D. et al. 2013. "Preparation of Activated Carbon Aerogels with Hierarchically Porous Structures for Electrical Double Layer Capacitors." Electrochimica Acta 89: 571-6.

[22] Liu, R. et al. 2016. "Nitrogen-Doped Carbon Microfiber with Wrinkled Surface for High Performance Supercapacitors." Scientific Reports 6: 21750.

[23] Yang, I. et al. 2016. "Pore Size-Controlled Carbon Aerogels for EDLC Electrodes in Organic Electrolytes." Current Applied Physics 16 (6): 665-72.

[24] Liew, C.-W., Ramesh, S., and Arof, A. K. 2016. "Investigation of Ionic Liquid-Doped Ion Conducting Polymer Electrolytes for Carbon-Based Electric Double Layer Capacitors (EDLCs).” Materials \& Design 92: 829-35.

[25] Wang, K. et al. "Nitrogen-Modified Biomass-Derived Cheese-Like Porous Carbon for Electric Double Layer Capacitors. " RSC Advances 6 (32): 26738-44.

[26] Mai, L.-Q. et al. 2013. "Synergistic Interaction between Redox-Active Electrolyte and Binder-Free Functionalized Carbon for Ultrahigh Supercapacitor Performance." Nat Commun 4.

[27] Huang, J., Sumpter, B. G., and Meunier, V. 2008. "A Universal Model for Nanoporous Carbon Supercapacitors Applicable to Diverse Pore Regimes, Carbon Materials, and Electrolytes." Chemistry - A European Journal 14 (22): 6614-26.

[28] Lu, Q., Chen, J. G., and Xiao, J. Q. 2013. "Nanostructured Electrodes for High-Performance Pseudocapacitors." 
Angewandte Chemie International Edition 52 (7): 1882-9.

[29] Zhi, J. et al. 2014. "Study on the Relation between Pore Size and Supercapacitance in Mesoporous Carbon Electrodes with Silica-Supported Carbon Nanomembranes." RSC Advances 4 (76): 40296-300.

[30] Choi, C. et al. 2016. "Elastomeric and Dynamic $\mathrm{MnO} 2 / \mathrm{CNT}$ Core-Shell Structure Coiled Yarn Supercapacitor." Advanced Energy Materials 6 (5): 1502119.

[31] Chen, H., Zhou, S., and Wu, L. 2014. "Porous Nickel Hydroxide-Manganese Dioxide-Reduced Graphene Oxide Ternary Hybrid Spheres as Excellent Supercapacitor Electrode Materials." ACS Applied Materials \& Interfaces 6 (11): 8621-30.

[32] Ghosh, D., Mandal, M., and Das, C. K. 2015. "Solid State Flexible Asymmetric Supercapacitor Based on Carbon Fiber Supported Hierarchical $\mathrm{Co}(\mathrm{OH}) \mathrm{xCO}_{3}$ and $\mathrm{Ni}(\mathrm{OH})_{2}$." Langmuir 31 (28): 7835-43.

[33] Wang, R. et al. 2016. "Ruthenium Oxide/Reduced Graphene Oxide Nanoribbon Composite and Its Excellent Rate Capability in Supercapacitor Application." Chinese Journal of Chemistry 34 (1): 114-22.

[34] Muniraj, V. K. A., Kamaja, C. K., and Shelke, M. V. 2016. " $\mathrm{RuO}_{2} \cdot \mathrm{nH}_{2} \mathrm{O}$ Nanoparticles Anchored on Carbon Nano-onions: An Efficient Electrode for Solid State Flexible Electrochemical Supercapacitor." ACS Sustainable Chemistry \& Engineering 4 (5): 2528-34.

[35] Yang, K. S., Kim, C. H., and Kim, B.-H. 2015. "Preparation and Electrochemical Properties of RuO2-Containing Activated Carbon Nanofiber Composites with Hollow Cores." Electrochimica Acta 174: 290-6.

[36] Wang, G. et al. 2015. "One-Step Synthesis of Mesoporous $\mathrm{MnO} 2 /$ Carbon Sphere Composites for Asymmetric Electrochemical Capacitors." Journal of Materials Chemistry A 3 (3): 1127-32.
[37] Huang, H. et al. 2015. "Controlled Growth of Nanostructured $\mathrm{MnO}_{2}$ on Carbon Nanotubes for High-Performance Electrochemical Capacitors." Electrochimica Acta 152: 480-8.

[38] Hsieh, C.-T. et al. 2016. "Deposition of $\mathrm{MnO} 2$ Nanoneedles on Carbon Nanotubes and Graphene Nanosheets as Electrode Materials for Electrochemical Capacitors." Journal of Alloys and Compounds 660: 99-107.

[39] Gambou-Bosca, A., and Bélanger, D. 2015. "Chemical Mapping and Electrochemical Performance of Manganese Dioxide/Activated Carbon Based Composite Electrode for Asymmetric Electrochemical Capacitor." Journal of The Electrochemical Society 162 (5): A5115-23.

[40] Liu, C. et al. 2015. "Coherent $\mathrm{Mn}_{3} \mathrm{O}_{4}$-Carbon Nanocomposites with Enhanced Energy-Storage Capacitance." Nano Research 8 (10): 3372-83.

[41] Jeong, G. H. et al. 2016. "Metal Oxide/Graphene Composites for Supercapacitive Electrode Materials." Chemistry-An Asian Journal 11 (7): 949-64.

[42] Caiying, C. et al. 2014. "NiO/Nanoporous Graphene Composites with Excellent Supercapacitive Performance Produced by Atomic Layer Deposition." Nanotechnology 25 (50): 504001.

[43] Jiang, W. et al. 2015. "Ternary Hybrids of Amorphous Nickel Hydroxide-Carbon Nanotube-Conducting Polymer for Supercapacitors with High Energy Density, Excellent Rate Capability, and Long Cycle Life." Advanced Functional Materials 25 (7): 1063-73.

[44] Zhou, J. et al. 2015. "Ultrahigh Volumetric Capacitance and Cyclic Stability of Fluorine and Nitrogen Co-doped Carbon Microspheres." Nat Commun 6: 8503.

[45] $\mathrm{Ma}$, W. et al. 2016. "Hierarchical $\mathrm{MnO}_{2}$ Nanowire/Graphene Hybrid Fibers with Excellent Electrochemical Performance for Flexible Solid-State Supercapacitors." Journal of Power Sources 306: 481-8. 